\title{
Laboratorios de logística internacional como modelo de competitividad aplicado en las universidades de Colombia
}

Laboratories of international logistics as a model of competitiveness applied in the universities of Colombia

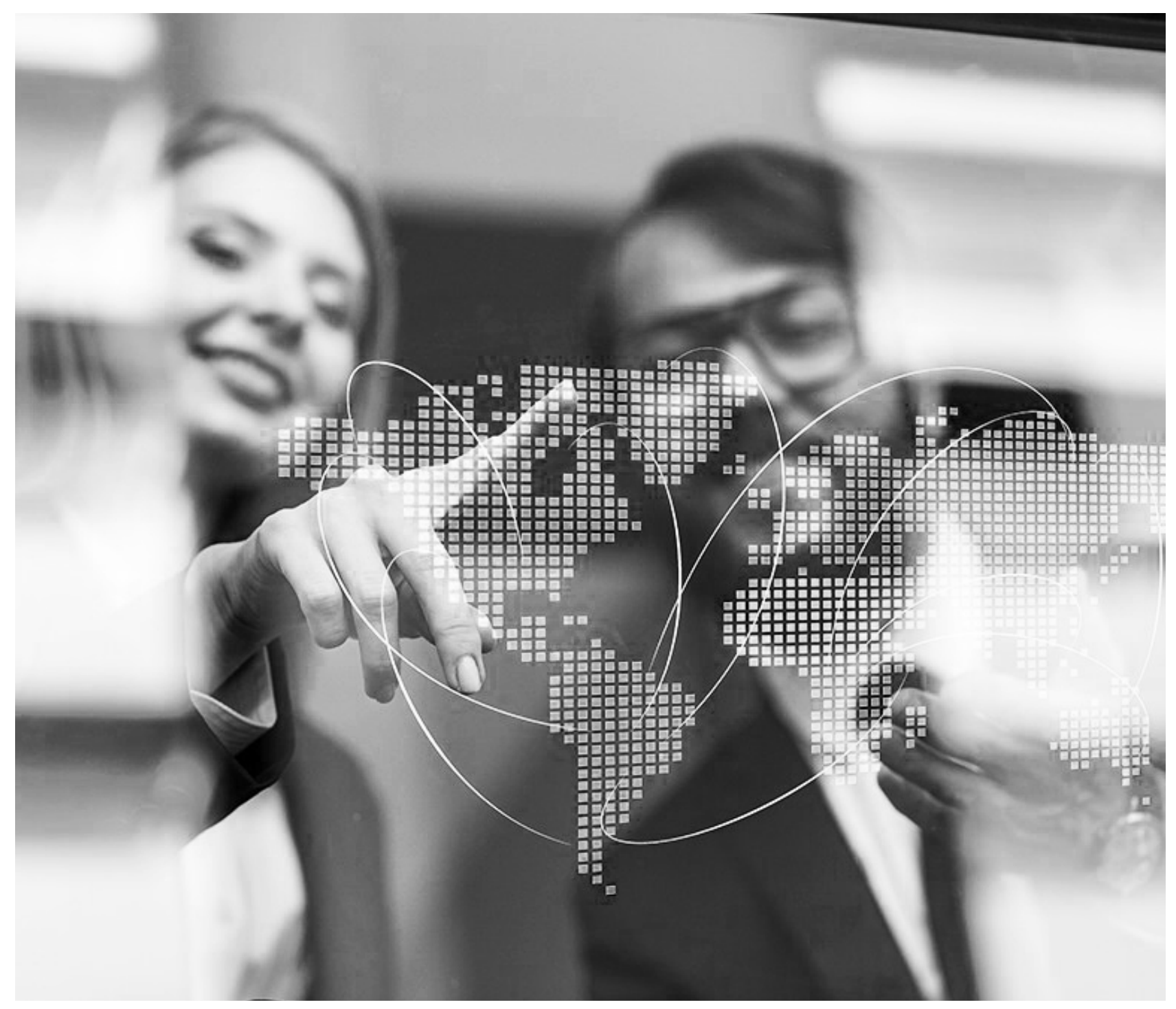




\title{
Laboratorios de logística internacional como modelo de competitividad aplicado en las universidades de Colombia ${ }^{1}$ Laboratories of international logistics as a model of competitiveness applied in the universities of Colombia
}

\author{
Maryan Natalia Castro Peña ${ }^{2}$, Luis Alfonso Robles Rangel ${ }^{3}$
}

Artículo recibido en abril de 2018; artículo aceptado en octubre de 2018.

Este artículo puede compartirse bajo la Licencia Creative Commons Atribución-NoComercial-Compartirlgual 4.0 Internacional y se referencia usando el siguiente formato: Castro, M. \& Robles, L. (2019). Laboratorios de logística internacional como modelo de competitividad aplicado en las universidades de Colombia. I+D Revista de Investigaciones, 13 (1), 106-116.

DOI: https://doi.org/10.33304/revinv.v13n1-2019009

\begin{abstract}
Resumen
En los últimos diez años se ha venido incrementando el número de universidades que cuentan con programas académicos relacionados con los negocios internacionales o el comercio exterior, esto debido a las necesidades que han surgido en el mercado laboral producto de la globalización. Asimismo, estos programas académicos se han consolidado y optimizado con los años, ya que estas mismas necesidades del mercado laboral llevan a nuevos y mejores conocimientos en el ámbito de los Negocios Internacionales, exigiendo mayor preparación, formación y aplicación de conocimiento en los futuros profesionales que tendrán a cargo actividades y responsabilidades en el área de comercio exterior de empresas nacionales e internacionales. Sin embargo, existe una prioridad en la formación de los negociadores internacionales y es el hecho de ser fundamentalmente operativos, lo que da lugar a procesos de enseñanza y aprendizaje basados en la aplicación y desarrollo de casos reales que le permitan al estudiante entrar en contacto con ciertas situaciones que puede experimentar en su mundo laboral real. Es precisamente allí donde asignaturas como Logística de la Distribución Física Internacional requieren de un espacio físico en el que se puedan aplicar y/o desarrollar los conocimientos e investigaciones adquiridas en las aulas, por lo cual se resalta la importancia de los laboratorios de logística internacional como apoyo al modelo pedagógico de cada institución de educación superior en esta área.
\end{abstract}

Palabras clave: logística internacional, competitividad, estrategias, enseñanza, aprendizaje.

\footnotetext{
Abstract

In the last ten years, the number of universities that have academic programs related to International Business or Foreign Trade has increased, this is due to the needs that have arisen in the labor market as a result of Globalization. Also, these academic programs have been consolidated and improved whit the years, as these same needs of

1. Artículo de investigación con enfoque cuantitativo. Resultado de un proyecto de investigación culminado, perteneciente al área de ciencias sociales, sub-área educación comercial y derecho. Fecha de inicio: febrero 2017. Fecha de finalización: febrero 2018.

2. Profesional en Comercio Exterior, Universidad Santo Tomás. Magíster en Tecnologías Aplicadas a la Educación, Universidad Manuela Beltrán. Docente de la Universidad de Santander - UDES (Bucaramanga, Colombia) Dirección: Calle 70 No. 55-210, PBX: (7) 6516500. ORCID ID: https://orcid. org/0000-0002-6627-8596.Correo electrónico: maryannataliacp@hotmail.com.

3. Profesional en Negocios Internacionales, Universidad Santo Tomás. Magíster en Dirección y Planificación de Empresas - MBA, Universidad Politécnica de Valencia. Magíster en Gestión de TI (Candidato), Universidad de Investigación y Desarrollo - UDI. Docente de la Universidad de Investigación y Desarrollo (Bucaramanga, Colombia). Dirección: Calle 9 No. 23-55, PBX: (57) (7) 635 2525. ORCID ID: https://orcid.org/0000-00015333-9567 Correo electrónico: roblesrangel@gmail.com.
} 
the labor markets lead to new and better knowledge in the field of International Business, demanding more preparation, training and application of knowledge in future professionals who will are in charge activities and responsibilities in the foreign trade area of national and international companies. However, there is a priority in the formation of International Negotiators and is the fact of being fundamentally operative, which gives rise to teaching and learning processes based on the application and development of real cases that, allow the student to come into contact with certain situations that they can experiment in their real working world. It is precisely here that subjects such as Logistics of International Physical Distribution require a physical space in which to apply and/ or develop the knowledge and research acquired in classrooms, which highlights the importance of laboratories in international logistics, as support for the pedagogical model of each Higher Education Institution in this area.

Keywords: international logistics, competitiveness, strategies, teaching, learning.

\section{Introducción}

Los laboratorios de logística internacional como modelo de competitividad aplicados a la educación universitaria son una actividad que viene desarrollándose desde hace algunos años en el mundo; para el caso de Sur América, países como Argentina, Uruguay, Brasil y Chile están a la vanguardia en estos modelos. En Europa, países como España, Alemania y Francia (Council of Supply Chain Management Professionals, 2017) ofrecen programas de pregrado y postgrado que aplican técnicas didácticas para la formación de sus estudiantes a través de la implementación de las nuevas tecnologías de la información, herramientas que apoyan el desarrollo de competencias específicas (Arenas, Pedraza \& Alarcón, 2014; Simanca, Porras, Garrido \& Hernández, 2017), a las que cada vez se les suman nuevos retos más complejos debido a la competitividad global. Las economías de países como China llevan varios años implementando modelos de competitividad en las universidades a través de laboratorios de logística de todo tipo, hecho que ha generado un alto número de proyectos exitosos a través de la logística, lo cual se evidencia en el "Logistic Performance Index" The World Bank (2017). "La serie Report sigue siendo la evaluación más completa de la competitividad a nivel mundial que evalúa el panorama de competitividad de 140 economías, proporcionando una visión de los factores que impulsan su productividad y prosperidad" (World Economic Forum, 2017). El tema de la competitividad requiere una especialización con un alto grado de conocimiento, factor determinante para el éxito de los proyectos logísticos.

De la Figura 1 se establece que el resultado del análisis de estudio de competitividad mundial parte de unos pilares básicos compuestos por variables como instituciones, infraestructura, entorno económico, salud y educación primaria, enseñanza superior y formación, eficiencia del mercado de bienes, eficiencia del mercado de trabajo, desarrollo del mercado financiero, preparación tecnológica, tamaño del mercado, sofisticación empresarial, y el último, innovación; cada uno de estos pilares establece un rango, una puntuación y una tendencia. El informe determina en su ranking de competitividad que Suiza es el país que tiene el mejor índice de competitividad, por lo tanto, ocupa el puesto número 1 a nivel mundial, seguido por Singapur, Estados Unidos y Alemania. En el puesto 61 de este ranking se sitúa Colombia. La escala de colores de blanco a gris representa el nivel de competencia alcanzado por cada una de las naciones, siendo los de color más oscuro los más competitivos.

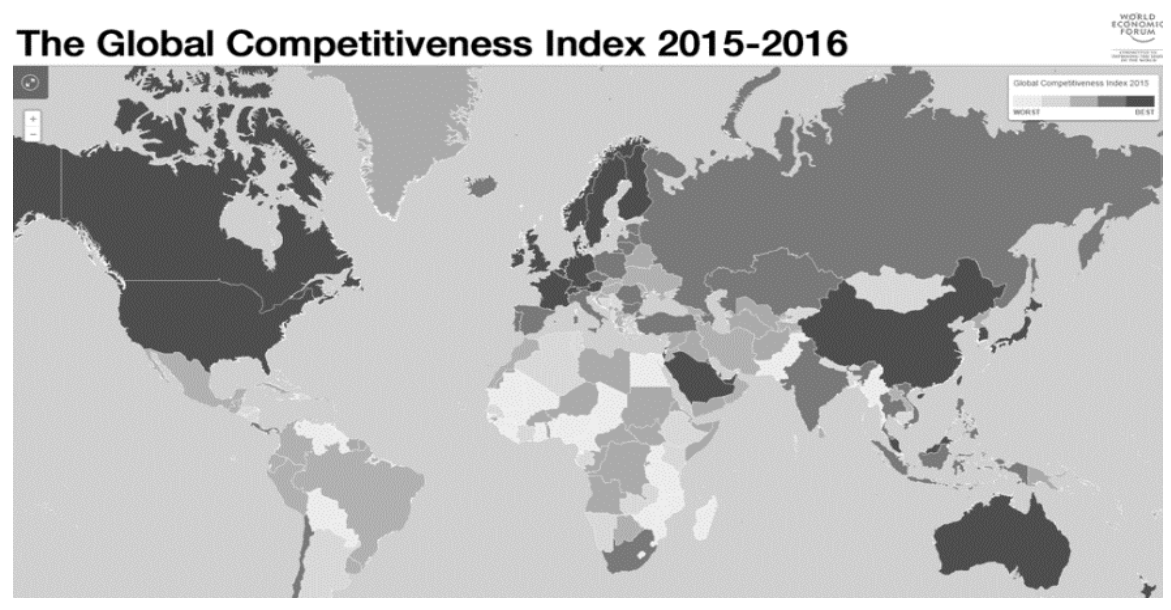

Figura 1. Competitividad Global. Fuente: World Economic Forum (2015 - 2016). 
De acuerdo con la tendencia de la competitividad mundial, el modelo por competencias analizado por el World Economic Forum (WEF) determina algunos pilares referentes a la educación, razón por la cual es necesario identificar para Colombia los aportes que realizan las instituciones educativas, tanto del sector público como privado, para el mejoramiento de estos indicadores. Desde la formación universitaria se transmiten conceptos que permiten adquirir conocimientos académicos basados en diferentes puntos de vista desde la perspectiva logística; entre estos conceptos se encuentra la Teoría de la Localización de Von Thunen (1826), que considera la ubicación geográfica como un elemento clave en las operaciones comerciales, centrando los polos de desarrollo en lugares con características geográficas de accesibilidad para facilitar el transporte, así como la posibilidad de manipular y acceder a otros mercados. Por su parte, Li (2007) en su libro Gestión de la cadena de suministro: conceptos, técnicas y prácticas mejora del valor mediante la colaboración, afirma que una adecuada gestión de los recursos y medios pertinentes para la administración y el control de los procesos de compras, almacenamiento y distribución -que pueden ser aplicados a la cadena de suministro-, permite establecen patrones que faciliten la generación de proyectos de envergadura para los diferentes sectores de la economía que involucren entes gubernamentales y privados. Esto, a su vez, genera oportunidades de eficiencia colaborativa en pro del desarrollo sostenible del Estado a través de políticas específicas aplicadas a los modelos de integración para la toma de decisiones.

Asimismo, Li (2007) y Von Thunen (1826) plantean soluciones a los problemas presentados a nivel internacional relacionados con la logística y su cadena de suministro global, como inventarios, compras, almacenaje, empaque y embalaje, unitarización, cubicaje, distribución física internacional, factor estiba, entre otros. En este escenario, las universidades se han convertido en un apoyo para el sector empresarial a través de la implementación de laboratorios logísticos en sus campus universitarios, cumpliendo un doble objetivo. Por una parte, los estudiantes ponen en práctica los conceptos teóricos adquiridos en las aulas de clases y, por otra, se estimula la interacción entre la academia y el sector real de la economía como motor de competitividad y crecimiento."Para la dirección y gestión de las organizaciones es indispensable centrarse en la logística, los enfoques aplicados a los procesos se encuentran en constante cambio y actualización debido a los cambios naturales de las economías mundiales" (Carrasco, 2000), visión que plantea una relación directa entre quienes desarrollan investigación logística, en este caso, las universidades, y quienes aplican esas investigaciones en las estructuras empresariales actuales. Este argumento es el que resalta la importancia de la creación de laboratorios de logística en las instituciones universitarias que ofertan carreras profesionales y afines.

El Estado colombiano cuenta con entidades que aplican como modelo el desarrollo de una formación enfocada a la práctica, cuyas instalaciones están dotadas con equipos con los cuales los aprendices se familiarizan con algunas de las herramientas que encontrarán en el desarrollo de sus actividades como técnicos y tecnólogos, ya sea trabajando para una compañía o en función de la creación de empresa por su propia cuenta, y hacen parte del programa denominado formación para el trabajo que se encuentra en el informe "Caracterización del sector de la logística en Colombia" elaborado por el Servicio Nacional de Aprendizaje [SENA] (2014).

Actualmente, el modelo es aplicado en algunas instituciones a nivel mundial, las cuales sustentan el Laboratorio de Producción y Logística - LPL -perteneciente a la Facultad de Ciencias Físicas y Matemáticas de la Universidad de Chile (2017), el cual "aplica los principios y métodos de la optimización matemática para generar soluciones a problemas industriales de alto impacto, como también para el apoyo en las decisiones de diseño o mejoramiento de políticas públicas".

La implementación de laboratorios logísticos en las instituciones de educación superior tiene como objetivo generar soluciones destinadas al sector empresarial, aportando valor agregado en el ámbito económico. Como evidencia de estas soluciones se encuentran los proyectos asociados a la Universidad de Chile, entre los cuales se destaca el desarrollado entre enero de 2005 y marzo de 2009, "Modelar matemáticamente el problema de las comunicaciones entre trenes en movimiento y de los trenes con las estaciones de control en tierra. Resultados: se diseñó y programó un sistema de simulación computacional orientado a trenes de alta velocidad (TGV) europeos" (Amaya, 2005). A través del laboratorio logístico se logró poner en marcha la integración de los sistemas ferroviarios de los países pertenecientes a la Unión Europea con la participación de los estudiantes y los docentes, quienes aportaron un conocimiento encaminado a generar una solución aplicable a los problemas logísticos del transporte ferroviario de carga y pasajeros utilizado en el viejo continente.

Para el caso de Colombia, algunas universidades cuentan con los laboratorios logísticos, como es el caso de la Pontificia Universidad Javeriana de Cali, la cual

(...) ofrece a la comunidad académica y empresarial el primer laboratorio universitario que integra 
el mercadeo, la logística y el consumo masivo. Diseñado con el apoyo técnico de GS1 Colombia (Logyca), este laboratorio incorpora tecnologías de punta, tales como videocámaras digitales para estudiar el comportamiento del consumidor y la góndola inteligente basada en la lectura de un código electrónico de los productos mediante radiofrecuencia (RFID), la cual permite realizar un seguimiento al producto desde que ingresa al centro de distribución y es comercializado en los puntos de venta. Toda esta gestión está soportada por un sistema de información empresarial integrado (ERP). (Pontificia Universidad Javeriana de Cali, 2017)

La implementación de los laboratorios logísticos en el marco de la educación universitaria potencia la función académica a niveles de cooperación e integración dinámica, permitiendo no solo ofrecer posibles soluciones a problemas del mercado en general, sino también incentivar a los estudiantes para aplicar el modelo de emprendimiento como una herramienta para el crecimiento económico y a aportar a la economía nacional.

Este caso no es el único en Colombia. La Universidad Autónoma del Occidente cuenta con diversos laboratorios que trabajan en conjunto para crear productos competitivos desde la academia, como el Laboratorio de Gestión de Operaciones, encargado de la sistematización de las líneas de producción para las pyme. Dicha sistematización permite agilizar los procesos internos minimizando el tiempo, reduciendo costos y mejorando la calidad. Por su parte, el Laboratorio de Procesos Industriales centra su actividad en el estudio de los materiales, su composición química y las posibles mejoras que puedan ser aplicadas en las diferentes empresas productivas del país. Asimismo, esta universidad cuenta con el Laboratorio de Logística, que tiene como objetivo formar a los estudiantes en las técnicas para la gestión de almacenamiento e inventarios, la creación de sistemas de transporte y distribución, y la aplicación de las tecnologías de la información y las comunicaciones en los entornos logísticos mundiales (Universidad Autónoma de Occidente, 2017).

Este hecho da cuenta de la intención del gobierno nacional colombiano, con el apoyo de las entidades del sector privado, de realizar un esfuerzo importante para la consecución de objetivos encaminados al mejoramiento de la competitividad nacional, entendiendo el sector educativo como base fundamental de un Estado o nación para la generación de la riqueza y el valor agregado implícito en sus actividades económicas.

Un reciente estudio, que emana del décimo Informe Nacional de Competitividad 2016 - 2017, elaborado por el Consejo Privado de Competitividad (2017), hace una radiografía de la evolución de la educación en Colombia en la última década y establece algunas recomendaciones para mejorar los niveles de competitividad nacional. Una de las recomendaciones presentadas es potenciar el involucramiento del sector empresarial en la academia, con un plazo para su ejecución fijado como corto, con actores definidos, entre los cuales se encuentran el Ministerio de Educación, las instituciones de educación superior y el sector empresarial, en tanto que el compromiso entre las diferentes instituciones públicas y privadas de participar activamente en las propuestas de mejora continua -como elemento diferenciador y necesario para un desarrollo equitativo y duradero- es el que permite soñar con una realidad diferente para las futuras generaciones.

\section{Metodología}

\section{Tipo de estudio}

La presente investigación se fundamenta en aplicar la logística internacional en los modelos educativos universitarios a través delaboratoriosyen establecerla percepción de los estudiantes frente a esta estrategia y/o modelo de competitividad.

La investigación propone la apertura de laboratorios para el desarrollo de las actividades de formación universitaria enfocadas en la logística internacional, esto, basados en el éxito que su implementación ha tenido en otros países. Para ello es indispensable analizar los resultados y los efectos que se pueden generar tras la implementación y uso de dichos laboratorios, y cómo ello puede fortalecer las estrategias de enseñanza y aprendizaje en la educación universitaria en Colombia. Dichos laboratorios se consideran una alternativa para que, antes de salir a aplicar sus conocimientos en el mundo real, los estudiantes realicen sus prácticas en estos espacios, donde se pueden realizar experimentos, investigaciones y proyectos.

De acuerdo con lo anterior, el enfoque de la investigación es cuantitativo, ya que es necesario medir tanto la necesidad de los laboratorios en las universidades con programas académicos de Negocios Internacionales como los resultados de la implementación de dichos laboratorios. Valga acotar que Gómez (2006) señala que, bajo la perspectiva cuantitativa, la recolección de datos es equivalente a medir.

El diseño de la presente investigación es experimental, ya que se eligió crear un laboratorio equipado con los elementos necesarios para que los estudiantes desarrollen allí actividades experimentales de logística internacional, para, a futuro, definir la eficiencia de la utilización del laboratorio. 
Puesto que la presente investigación se basa en un diseño cuasiexperimental, durante el proceso investigativo se manipulará una variable independiente (Laboratorio de Logística Internacional) y después se observarán las consecuencias que dicha manipulación tuvo sobre la variable dependiente (Universidades de Colombia). Valga anotar que "Los diseños cuasi experimentales también manipulan deliberadamente, al menos, una variable independiente para observar su efecto y relación con una o más variables dependientes..." (Hernández, Fernández \& Baptista, 2010).

Las variables de la investigación son:

- Variable independiente: Laboratorio de Logística Internacional

- Variable dependiente: Universidades de Colombia

Ahora bien, la investigación que se realizará es longitudinal o evolutiva, ya que la finalidad es analizar si el desarrollo de laboratorios de logística internacional aporta beneficios reales a la comunidad educativa de las universidades de Colombia. Esto se logra a través de la recolección de datos durante periodos definidos para, posteriormente, concluir si se presentaron cambios positivos o negativos y las consecuencias de estos.

\section{Participantes}

La población está estipulada por sus características definitorias, es decir, la población sería el conjunto de individuos de la misma clase delimitada por el estudio. Según Tamayo (1997), "La población se define como la totalidad del fenómeno a estudiar donde las unidades de población poseen una característica común la cual se estudia y da origen a los datos de la investigación".

La población de la presente investigación corresponde a las universidades de la ciudad de Bucaramanga, en el Departamento de Santander - Colombia, debido a que la implementación de estos laboratorios logísticos tiene unos altos costos para incorporarlos en las universidades. El presente artículo pretende demostrar la importancia que tienen estos laboratorios, y en el supuesto de que se logrará desarrollar una prueba que permita obtener resultados directos, se espera que se tenga en cuenta la ciudad mencionada, ya que los investigadores se encuentran allí ubicados, lo que facilita su aplicación. Además, sería importante iniciar en una ciudad de Colombia donde no se haya implementado aún la idea de la presente investigación. Para analizar los futuros resultados de la investigación se define como muestra los estudiantes de la asignatura de Logística Internacional o Distribución Física Internacional de las diferentes universidades de Bucaramanga, Santander; es importante resaltar que las universidades deben tener dentro de sus programas de formación profesional el programa de Negocios Internacionales o programas afines. Las características de la población a estudiar son:

\section{Población:}

- Universidades de Bucaramanga - Santander que cuentan con el Programa de Negocios Internacionales. El número de individuos que reúnen dichas características es tres (3). - Estudiantes del Programa de Negocios Internacionales o afines de las universidades de Bucaramanga- Santander: estudiantes en las asignaturas de Logística Internacional - Distribución Física Internacional. La población que será estudiada, para cada universidad, se distribuye así:

- Universidad Autónoma de Bucaramanga - UNAB: 323 estudiantes.

- Universidad Santo Tomás - USTA: 205 estudiantes.

- Universidad de Investigación y Desarrollo - UDI: 185 estudiantes.

- La edad de los estudiantes es de entre 20 y 35 años.

- Los estudiantes son mujeres y hombres. Se estima que la población es de aproximadamente 20 a 25 estudiantes en cada universidad, debido a que las asignaturas como Logística Internacional y afines se cursan en los últimos semestres de los programas académicos.

El muestreo es no probabilístico intencional, donde se eligen los individuos que se estima que son representativos o típicos de la población. Siguiendo el criterio del experto o el investigador se seleccionan los sujetos que se estima puedan facilitar la información necesaria (Hernández, Fernández \& Baptista, 2010), y se clasifican así:

Muestra 1: Está conformada por todas las universidades de Bucaramanga que cuentan con el programa académico Negocios Internacionales o afines.

Muestra 2: Está conformada por los estudiantes de las asignaturas de Logística Internacional o Distribución Física Internacional.

\section{Materiales e instrumentos}

Los instrumentos de recolección de datos son claves para poder definir y clasificar las observaciones y los resultados. Para hacer una adecuada recolección de datos es importante emplear los instrumentos indicados, pues dichos instrumentos permitirán registrar datos observables que indiquen verdaderamente los conceptos - las variables que se necesitan en esta investigación (Grinnell, Unrau, \& Williams, 2009). 
Las fuentes de donde se obtendrán lo datos serán suministradas por los docentes y por los estudiantes de los Programa de Negocios Internacionales de las Universidades de Bucaramanga, utilizando técnicas de encuesta y observación de aula.

Las técnicas y los instrumentos que se emplearán se definen así:

\section{Técnica: Observación}

Instrumento: Guía de observación en el aula

Técnica: Encuesta

Instrumento: Cuestionario

\section{Encuesta}

Con la encuesta como técnica de recolección de datos para la presente investigación, se desarrolló un cuestionario compuesto por diez preguntas con opción de múltiple respuesta, que fue enviado a los participantes de la investigación a través de la herramienta Google forms, la cual brinda la libertad diligenciar dichos cuestionarios dentro y fuera de las universidades. El cuestionario fue elaborado por los investigadores, y el enlace fue enviado vía correo electrónico a cada estudiante.

Las preguntas que componen el cuestionario se relacionan en la sección Resultados del presente documento.

\section{Observación natural}

Mediante actividades de campo se realizó un recorrido por las aulas de clase de las tres universidades partícipes de la investigación, tomando como evidencia fotografías del estado actual de los lugares donde se imparten las clases de Logística Internacional (ver Figuras 2, 3 y 4).

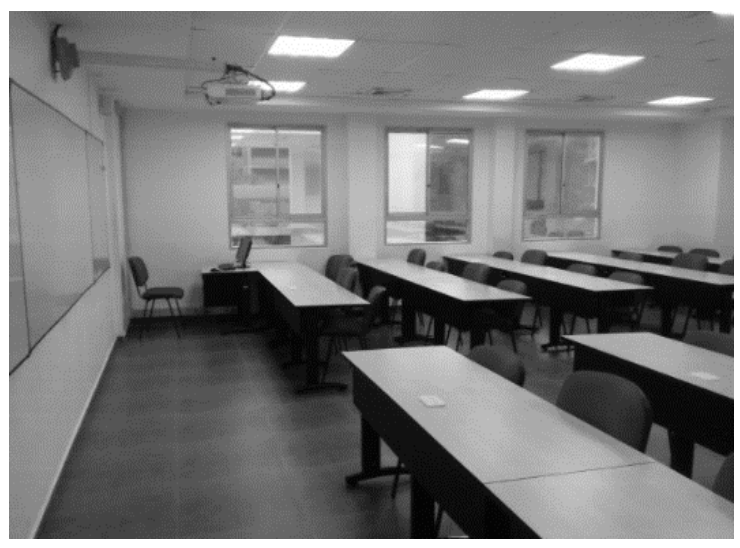

Figura 2. Aula de clase en la Universidad Autónoma de Bucaramanga, UNAB. Fuente: Autores.

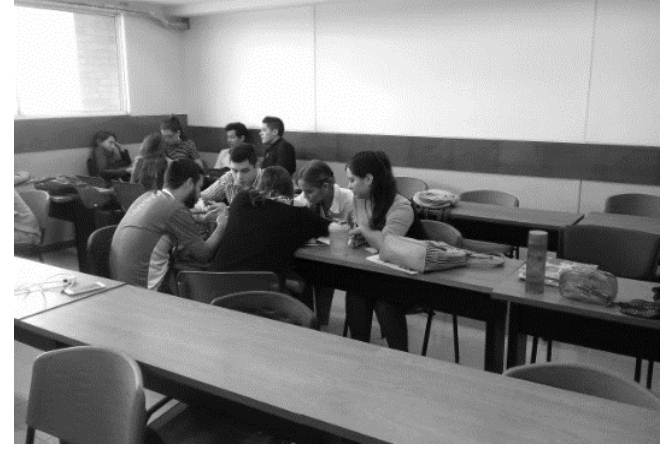

Figura 3. Aula de clase en la Universidad de Investigación y Desarrollo, UDI. Fuente: Autores.

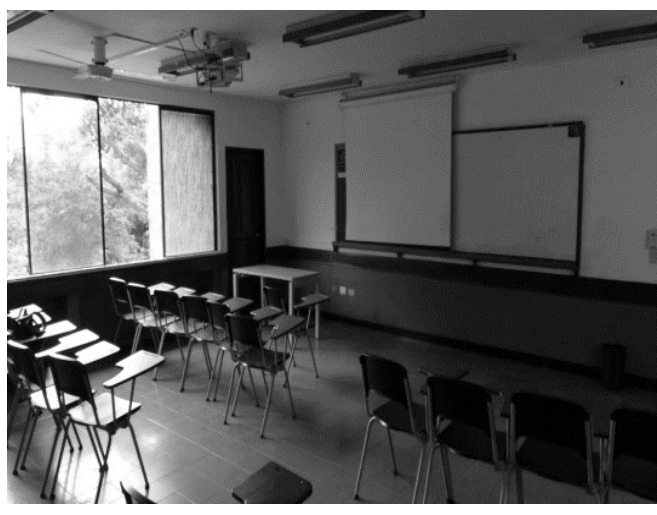

Figura 4. Aula de clase en la Universidad Santo Tomás, USTA. Fuente: Autores.

\section{Procedimiento}

Los pasos a seguir para el desarrollo de la presente investigación se detallan a continuación:

- Identificar las universidades de Bucaramanga, que cuentan con el programa académico de Negocios Internacionales o programas afines de formación profesional.

- Establecer contacto con las respectivas direcciones de programa de las universidades para solicitar autorización con el fin de desarrollar la investigación.

- Iniciar con la implementación del Instrumento de Guía de observación en el aula en las asignaturas de Logística Internacional y/o Distribución Física Internacional en dichas universidades.

- Elaborar el cuestionario de preguntas para desarrollar en línea.

- Analizar y tabular los resultados.

- Determinar la aceptación del tema propuesto de investigación en la comunidad estudiantil.

- A partir de los resultados obtenidos se proponen algunos comentarios y recomendaciones para las instituciones de educación superior - IES, en lo referente a la incorporación de laboratorios de logística internacional. 
En Colombia aún no se observan resultados de la aplicación de laboratorios logísticos tan específicos como los señalados en el presente artículo; sin embargo, alrededor del mundo sí hay evidencias dela aplicación de laboratorios de logística con otro componente específico. Un ejemplo de los laboratorios de logística implementados en las universidades del mundo que han aportado un valor agregado a la educación de los estudiantes, es el Laboratorio de Logística de la Universidad de Dortmund, Alemania, implementado en el año 2007, gracias a los esfuerzos de los doctores Axel Kuhn, profesor de Flujo de Materiales y Logística del Instituto Fraunhofer, ErnstHermann Krog, jefe de logística de la marca Audi, y Roland Kischkel, rector de la Universidad de Dortmund. Dicha iniciativa se concretó a través de la creación de Escuela de Producción de Ingeniería Logística, con un presupuesto de $500.000 €$, con el objetivo principal de fortalecer la sinergia entre la academia y la empresa privada para permitir que los estudiantes de postgrado (doctorado) trabajen (durante tres años) en la generación de proyectos de investigación que permitan aumentar la eficiencia y generar innovaciones como un pilar esencial de efectividad logística para la marca Audi y sus automóviles.

Otro ejemplo de los buenos resultados de los laboratorios de logística es el proyecto "Investigación y construcción del sistema de ingeniería de laboratorio de logística moderna de la Universidad de Tecnología de Xi'an", promovido por docentes chinos. A través de la implementación de laboratorios de logística en diferentes universidades nacionales de China se logró la construcción de un sistema moderno de ingeniería integral, innovadory abierto, basado en los antecedentes de la ingeniería de fabricación; este sistema satisface los requisitos de enseñanza actual y la investigación (Yang, Shi \& Li, 2012; Yang et al., 2008).

\section{Resultados}

Para identificar la situación actual de la implementación de laboratorios de logística en las universidades de la ciudad de Bucaramanga, se aplicó una encuesta en línea a los estudiantes de las tres universidades en las que se desarrolla el programa académico profesional en Negocios Internacionales, a saber: Universidad Autónoma de Bucaramanga - UNAB, Universidad de Investigación y Desarrollo - UDI y Universidad Santo Tomás - USTA.

Los estudiantes que están actualmente cursando la asignatura de Logística Internacional en estas tres universidades suman un total de 97 , repartidos de la siguiente manera:

UNAB: 35 estudiantes. UDI: 40 estudiantes.

USTA: 22 estudiantes.
El cuestionario fue enviado a la totalidad deestos estudiantes, de los cuales 89 atendieron y respondieron la encuesta.

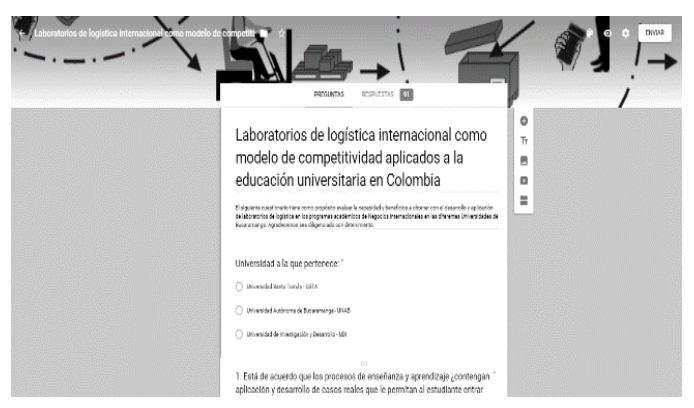

Figura 5. Encuesta aplicada. Fuente: Autores. Disponible en: https://goo.gl/forms/3Y26WmHupoUSRyYp2

A continuación, se detallan los resultados obtenidos:

1. Está de acuerdo con que los procesos de enseñanza y aprendizaje contengan aplicación y desarrollo de casos reales que le permitan al estudiante entrar en contacto con ciertas situaciones que pueden experimentar en su mundo real laboral?

\section{Tabla 1}

Resultado de la pregunta uno

\begin{tabular}{lc}
\hline \multicolumn{1}{c}{ Opciones } & Respuestas (porcentaje) \\
\hline Totalmente de acuerdo & $81.3 \%$ \\
De acuerdo & $15.4 \%$ \\
Indiferente & $1.1 \%$ \\
En desacuerdo & $1.1 \%$ \\
Totalmente en desacuerdo & $1.1 \%$ \\
\hline
\end{tabular}

Fuente: Autores.

2. ¿Considera que la asignatura Logística y Distribución Física Internacional - DFI requiere de un espacio adecuado en el que se puedan aplicar y/o desarrollar las investigaciones y conocimientos adquiridos en las aulas de forma práctica?

\section{Tabla 2}

\section{Resultado de la pregunta dos}

\begin{tabular}{lc}
\hline \multicolumn{1}{c}{ Opciones } & Respuestas \% \\
\hline SI & $96.7 \%$ \\
NO & $1.1 \%$ \\
Logística y Distribución Física Internacional & $1.1 \%$ \\
Visitas técnicas a compañías & $1.1 \%$ \\
\hline
\end{tabular}

Fuente: Autores. 
3. ¿Conoce o ha escuchado de laboratorios logísticos implementados en Universidades de Colombia?

\section{Tabla 3}

\section{Resultado de la pregunta tres}

\begin{tabular}{lc}
\hline \multicolumn{1}{c}{ Opciones } & Respuestas \% \\
\hline Ha escuchado & $33 \%$ \\
Ha escuchado y conoce & $5.5 \%$ \\
Los conoce & $3.3 \%$ \\
Ni ha escuchado ni los conoce & $58.2 \%$ \\
\hline
\end{tabular}

Fuente: Autores.

4. En Colombia hay varias universidades que cuentan con laboratorios logísticos aplicados como apoyo al modelo educativo, entre ellas la Pontificia Universidad Javeriana de Cali y la Universidad Autónoma del Occidente de la misma ciudad. ¿Considera que las universidades de Bucaramanga deberían sumarse a este reto?

Tabla 4

Resultado de la pregunta cuatro

\begin{tabular}{lc}
\hline \multicolumn{1}{c}{ Opciones } & Respuestas \% \\
\hline $\begin{array}{l}\text { Es un espacio de aplicación de conocimiento e } \\
\text { interacción. }\end{array}$ & $48.4 \%$ \\
$\begin{array}{l}\text { Es una oportunidad de mejorar las estrategias de } \\
\text { enseñanza y aprendizaje. }\end{array}$ & $63.7 \%$ \\
$\begin{array}{l}\text { Es una pérdida de tiempo y dinero. } \\
\text { Aún no estamos preparados para ese tipo de }\end{array}$ & $2.2 \%$ \\
actividades en la educación. & $2.2 \%$ \\
Me es indiferente & $2.2 \%$ \\
Otra & $3.3 \%$ \\
\hline
\end{tabular}

Fuente: Autores.

5. Basados en el éxito arrojado en la implementación de laboratorios logísticos en universidades de Colombia y del mundo, ¿apoyaría la iniciativa de desarrollarlas en las demás universidades de Colombia?

\section{Tabla 5}

Resultado de la pregunta cinco

\begin{tabular}{lc}
\hline \multicolumn{1}{c}{ Opciones } & Respuestas \% \\
\hline Totalmente de acuerdo & $79.1 \%$ \\
De acuerdo & $16.5 \%$ \\
Indiferente & $4.4 \%$
\end{tabular}

\begin{tabular}{ll} 
En desacuerdo & $0 \%$ \\
Totalmente en desacuerdo & $0 \%$ \\
\hline
\end{tabular}

Fuente: Autores.

6. Los objetivos que cumple la implementación de laboratorios logísticos en los campus universitarios son: 1-Realizarla práctica de los conceptos teóricos adquiridos en las aulas de clase, 2Estimular la interacción entre la academia y el sector real de la economía como motor de competitividad y crecimiento. ¿Le gustaría que fueran incorporados en su universidad? Califique de 1 a 5 (1 menor gusto o 5 mayor gusto).

\section{Tabla 6}

\section{Resultado de la pregunta seis}

\begin{tabular}{cc}
\hline Opciones & Respuestas \% \\
\hline 1 & $1.1 \%$ \\
2 & $1.1 \%$ \\
3 & $3.3 \%$ \\
4 & $8.9 \%$ \\
5 & $85.6 \%$ \\
\hline
\end{tabular}

Fuente: Autores.

7. De acuerdo a su respuesta anterior seleccione la que más se ajusta a su criterio:

\section{Tabla 7}

Resultado de la pregunta siete

\begin{tabular}{lc}
\hline \multicolumn{1}{c}{ Opciones } & Respuestas \% \\
\hline Totalmente de acuerdo & $80.2 \%$ \\
De acuerdo & $17.6 \%$ \\
Indiferente & $1.1 \%$ \\
En desacuerdo & $1.1 \%$ \\
Totalmente en desacuerdo & $0 \%$ \\
\hline
\end{tabular}

Fuente: Autores.

8. ¿Considera que un espacio para realizar investigación experimental y pruebas logísticas apunta a fortalecer su perfil profesional en una escala porcentual de?

\section{Tabla 8}

Resultado de la pregunta ocho

\begin{tabular}{cc}
\hline Opciones & Respuestas \% \\
\hline $0 \%$ & $2.2 \%$ \\
$10 \%$ & $0 \%$
\end{tabular}




\begin{tabular}{cc}
$20 \%$ & $0 \%$ \\
$30 \%$ & $0 \%$ \\
$40 \%$ & $0 \%$ \\
$50 \%$ & $4.4 \%$ \\
$60 \%$ & $2.2 \%$ \\
$70 \%$ & $5.5 \%$ \\
$80 \%$ & $18.7 \%$ \\
$90 \%$ & $11 \%$ \\
$100 \%$ & $54.9 \%$ \\
\hline
\end{tabular}

Fuente: Autores.

9. ¿Está de acuerdo en que este modelo de enseñanza y aprendizaje permitirá que la educación universitaria en Colombia sea más competitiva?

\section{Tabla 9}

\section{Resultado de la pregunta nueve}

\begin{tabular}{lc}
\hline \multicolumn{1}{c}{ Opciones } & Respuestas \% \\
\hline Totalmente de acuerdo & $73.6 \%$ \\
De acuerdo & $23.1 \%$ \\
Indiferente & $2.2 \%$ \\
En desacuerdo & $1.1 \%$ \\
Totalmente en desacuerdo & $0 \%$ \\
\hline
\end{tabular}

Fuente: Autores.

10. ¿Considera que la integración de los laboratorios logísticos en el modelo pedagógico de su universidad le permitirá ingresar al mercado laboral más preparado?

\section{Tabla 10}

\section{Resultado de la pregunta diez}

\begin{tabular}{lc}
\hline \multicolumn{1}{c}{ Opciones } & Respuestas \% \\
\hline Totalmente de acuerdo & $72.2 \%$ \\
De acuerdo & $18.9 \%$ \\
Indiferente & $6.7 \%$ \\
En desacuerdo & $2.2 \%$ \\
Totalmente en desacuerdo & $0 \%$ \\
\hline
\end{tabular}

Fuente: Autores.

De acuerdo a las respuestas de los estudiantes en las encuestas realizadas, se evidencian los siguientes resultados:
Los alumnos de las tres universidades encuestadas coinciden en estar totalmente de acuerdo en la necesidad de aplicar y solucionar casos reales en su proceso de formación académica, por lo que se requiere disponer de un espacio adecuado para desarrollar actividades de investigación y prácticas de logística internacional. Pese a que la mayoría no conocen ni han escuchado de la implementación de laboratorios logísticos en las universidades, están totalmente de acuerdo en que las universidades de Bucaramanga deben seguir los pasos de aquellas que ya disponen de estos laboratorios en Colombia, y están totalmente de acuerdo en apoyar la iniciativa. Asimismo, a la mayoría los estudiantes encuestados les gustaría que existieran laboratorios de logística en su universidad, como se evidencia en la calificación de 5 en su nivel de gusto, ya que consideran que estos son espacios para la aplicación de conocimiento e interacción. Además, consideran que los laboratorios fortalecen su perfil profesional, y que estos índices contribuirán para que la educación universitaria en Colombia sea más competitiva, permitiendo a los estudiantes ingresar al mercado laboral mejor preparados.

\section{Comentarios}

La importancia de la logística en todas las actividades de la sociedad es innegable. Por su parte, los laboratorios de logística juegan un papel muy importante en las universidades a nivel mundial, puesto que permiten llevar el conocimiento teórico ala práctica, con el objetivo de preparar, desde la academia, profesionales en logística internacional que cumplan con los requisitos de la sociedad respecto a un perfil y unas competencias definidos. Al comparar los diferentes laboratorios de logística implementados en las universidades tanto nacionales como internacionales, se observó su amplia diversidad: algunos tienen por objetivo realizar experimentos; la visión de otros es incluir recursos de software y hardware; otros más buscan generar modelos que sean aplicados en las empresas y algunos más pretenden generar proyectos de impacto social, ambiental y económico. Lo que es importante destacar es que todos aportan para la generación de nuevo conocimiento y competitividad en el entorno globalizado actual.

\section{Recomendaciones}

La competitividad es un factor que determina el éxito de una organización, por ello, en la educación universitaria es necesario implementar estrategias pedagógicas que faciliten el desarrollo de competencias profesionales logísticas en los estudiantes, aplicando la enseñanza situada y el aprendizaje por experiencia a través de la metodología "aprender haciendo y enseñar demostrando", la cual le permite al estudiante construir su propio conocimiento, desarrollar 
habilidades y reforzar sus valores directamente desde la experiencia con la práctica productiva. Para las universidades, la posibilidad de analizar los resultados de los laboratorios a nivel mundial a partir del modelo de competitividad les permitirá afrontar los futuros retos de la economía y generar un modelo educativo con diferenciación y valor agregado.

Se sugiere estudiar la posibilidad de crear e implementar los laboratorios de logística internacional en las universidades de Bucaramanga que cuenten con el programa de Negocios Internacionales, con un estudio previo de presupuesto de acuerdo al PEI de cada institución.

Es necesario que las universidades sepan que los laboratorios de logística serán transversales para otros programas de la misma universidad, y que por ser una novedad dentro del sistema académico en la ciudad, las universidades que cuenten con dichos laboratorios generarán valor agregado en el programa académico $y$, por ende, reconocimiento académico a la institución.

\section{Agradecimientos}

La realización de esta investigación fue posible gracias a la colaboración de las diferentes universidades de la ciudad de Bucaramanga, de los directores de los programas de Negocios Internacionales, al apoyo del cuerpo docente y a la participación de los estudiantes de dichos programas. El apoyo recibido permitió la recolección de los datos de fuentes primarias y la documentación del proceso para la elaboración del presente artículo.

\section{Referencias}

Amaya, J. (2005). INTEGRAIL: "Intelligent Integration of Railway Systems", WP3D_05. Contract PL 012526, Programa Marco, Unión Europea.

Arenas, J. S., Pedraza, J. M. \& Alarcón, C. A. (2014). Ecosistema Digital Académico: Hacia una comunidad digital soportada en TIC para las instituciones de educación superior. I+D Revista de Investigaciones, 4(2), 6-14.

Carrasco, J. (2000). Evolución de los enfoques y conceptos de la logística: Su impacto en la dirección y gestión de las organizaciones. Economía Industrial, (331), 17-34.

Consejo Privado de Competitividad. (2017). Informe Nacional de Competitividad 2016-2017. Recuperado de https://compite.com.co/informe/informe-nacional-decompetitividad-2016-2017/

Council of Supply Chain Management Professionals. (2017). Educating and Connecting the World's Supply Chain Professionals. Recuperado de https://cscmp. org/

Gómez, M. M. (2006). Introducción a la Metodología de la Investigación Científica. Córdoba, Argentina: Editorial Brujas.

Grinnell, R., Unrau, Y. \& Williams, M. (2009). Research methods for BSW students. Pair Bond Publications.

Hernández, R., Fernández, C., \& Baptista, P. (2010). Metodología de la investigación (5a.ed.). (Interamericana Ed.): McGraw-Hill.

Li, L. (2007). Supply chain management: Concepts, techniques and practices: Enhancing value through collaboration. World scientific publishing company.

Pontificia Universidad Javeriana de Cali. (2017). Laboratorio de logistica y mercadeo. Recuperado de https://www.javerianacali.edu.co/laboratorios/ laboratorio-de-logistica-y-mercadeo

Servicio Nacional de Aprendizaje [SENA]. (2014). Caracterización del sector de la logística en Colombia. Recuperado de http://www.ciatijfk.org/ciatiweb/ images/publicapdf/8.

Simanca, F. A., Porras, A. A., Garrido, F. B. \& Hernández, P. C. (2017). Implementación de herramientas tecnológicas en los procesos de enseñanza- aprendizaje de los triángulos. I+D Revista de Investigaciones, 10(2), 71-79.

Tamayo, M. (1997). El proceso de la investigación científica (4a. ed.). México-España-Venezuela-Colombia: Limusa Noriega Editores.

The World Bank. (2017). Logistic performance index. Recuperado de https://lpi.worldbank.org/

Universidad Autónoma de Occidente. (2017). Laboratorios de Ingeniería Industrial. Recuperado de http://www.uao.edu.co/ingenieria/laboratoriosingenieria-industrial

Universidad de Chile. (2017). Laboratorio de Producción y Logística (LPL). Recuperado de http://ingenieria.uchile. $\mathrm{cl} /$ investigacion/presentacion/laboratorios/centro-demodelamiento-matematico/91342/laboratorio-deproduccion-y-logistica-Ipl

Von Thunen, J. H. (1826). The Isolated State. London: Pergamon Press.

World Economic Forum. (2017). The Global Competitiveness Report 2015-2016. Recuperado de http://reports.weforum.org/global-competitivenessreport-2015-2016/

Yang, H., Shi, C.-Q. \& Li, L. (2012). Comparison of Construction of Logistics Laboratories in Universities [J]. Research and Exploration in Laboratory, 10, 052.

Yang, S.-Q., Yuan, D.-N., Li, D.-X., Yang, J., Wang, W., Xie, J. \& Fu, W. (2008). Research and Construction of Modern Logistics Laboratory Engineering System [J]. Industrial Engineering Journal, 1, 034. 